\title{
ACROSTUDY: the first 5 years
}

\author{
Peter J Trainer \\ Department of Endocrinology, Christie Hospital, Wilmslow Road, Manchester M2O 4BX, UK \\ (Correspondence should be addressed to P J Trainer; Email: peter.trainer@manchester.ac.uk)
}

\begin{abstract}
ACROSTUDY is an observational registry intended to collect safety and efficacy data on pegvisomant therapy. A total of 792 patients have been enrolled, of whom $83 \%$ had commenced pegvisomant prior to recruitment. The mean follow-up is $\mathbf{1 . 6 6}$ years with the mean duration of pegvisomant therapy 3.31 years representing 2625 patient years of treatment. About $90 \%$ of patients were on once daily pegvisomant, and $67 \%$ were on monotherapy. Disappointingly, IGF1 was normalised in $<70 \%$ of patients; furthermore, in $80 \%$ of patients with an elevated IGF1, the daily dose of pegvisomant was $20 \mathrm{mg}$ or less. A total of 56 serious adverse events (AEs) were reported, of which 13 were related to pegvisomant. A total of 276 AEs were reported, of which 56 were considered related to pegvisomant. The AEs most frequently attributed to pegvisomant were disturbed liver function tests and injection site reactions. Magnetic resonance imaging (MRI) was available in 684 patients. A total of 411 patients had at least one MRI on pegvisomant compared with a baseline. In 31 patients, a decrease in tumour size has been reported, of whom 20 had previously received radiotherapy. An increase in tumour size has been reported and confirmed in 22 patients. In 11 patients, there was contradictory data on tumour size, while, in six patients, central review of the films failed to confirm increase in tumour size. In conclusion, the safety data are generally reassuring, while the IGF1 normalisation rate is disappointing, which probably reflects a failure of dose titration. Further effort is needed to understand the reasons for the failure of dose titration.
\end{abstract}

European Journal of Endocrinology 161 S19-S24

\section{Introduction}

Pegvisomant has been licensed in Europe since 2003 for the treatment of patients refractory to or intolerant of somatostatin analogues. Approximately, only 200 patients participated in the pre-registration clinical trials that demonstrated the ability of pegvisomant to relieve symptoms and normalise insulin-like growth factor 1 (IGF1) in virtually every patient $(1,2)$. The size and duration of the studies permitted only limited collection of safety data and, at the time of licensing, it was recognised that there was a need to continue collecting long-term experience of patients treated with this new class of drug. In contrast to all other modes of therapy for acromegaly, the mode of action of pegvisomant is not directed at the pituitary and does not endeavour to lower GH secretion, which actually increases (3). Before pegvisomant first entered clinical trials, there was concern that this novel mode of action carried the risk of expansion of pituitary tumours, with some speculation that inhibition of GH action could induce tumour growth in a manner analogous to the

This paper forms part of a special issue on $\mathrm{KIMS}^{\circledR}$ and ACROSTUDY $^{\mathrm{TM}}$. Pfizer Inc. has supported the publication of this special issue. growth of ACTH-secreting pituitary adenomas in Nelson's syndrome. In the pre-registration clinical trials, MRI scans were undertaken on at least a 6-monthly basis and were centrally reviewed, the data being essentially reassuring (4), although relatively short term. An unforeseen adverse event (AE) encountered during the early trials, and which is probably drug related, was disturbance of liver function tests, in particular elevation of alanine transaminase (ALT) and aspartate transaminase (AST). Although there have been a series of reports, including histology, the mechanism and consequences of the elevated liver function tests are uncertain (5-7). The need to collect safety and efficacy data and to understand how the drug is used outside the constraints of proscriptive clinical trials led to a desire for a post-launch prospective registry of patients on pegvisomant.

The first experience from such a registry has come from a German national database, which, since 2003, has gathered data on over 300 patients and provided valuable insight into the use of the drug in Germany and contributed to the development of an international registry $(8,9)$. ACROSTUDY is the international observational surveillance registry, sponsored by Pfizer, designed to gather safety and efficacy data on patients treated with pegvisomant (10). It is open to all patients 
on pegvisomant and, to date, 300 centres in 10 countries are participating. Data entry is web-based with central IGF1 measurement and review of pituitary imaging being offered.

This paper reports the experience from the first 5 years of data collected in ACROSTUDY.

\section{Materials and methods}

The first patient entered ACROSTUDY in 2004 and, at data freeze (February 2009), 792 patients (390 female, mean age 51.3 years) had been recruited. A total of 387 patients are known to have had surgery alone, 19 had radiotherapy alone and 241 had both surgery and radiotherapy prior to entering ACROSTUDY. It is important to appreciate that $83 \%$ of patients were already receiving pegvisomant at recruitment into ACROSTUDY.

Local ethical committee approval was obtained for each centre, and all patients have provided written informed consent.

The liver function tests, IGF1 values and reference ranges presented are from local laboratories and, similarly, the pituitary MR data are based on locally reported films unless otherwise stated.

\section{Results}

At data freeze (February 2009), the mean duration of follow-up within ACROSTUDY was 1.66 years, but as most patients had commenced pegvisomant before entry into ACROSTUDY, the mean duration of therapy was 3.31 years with the maximum duration of treatment of 10.38 years, amounting to an accumulative experience of 2625 patient years. At recruitment, $90 \%$ of patients entering ACROSTUDY were on oncedaily pegvisomant.

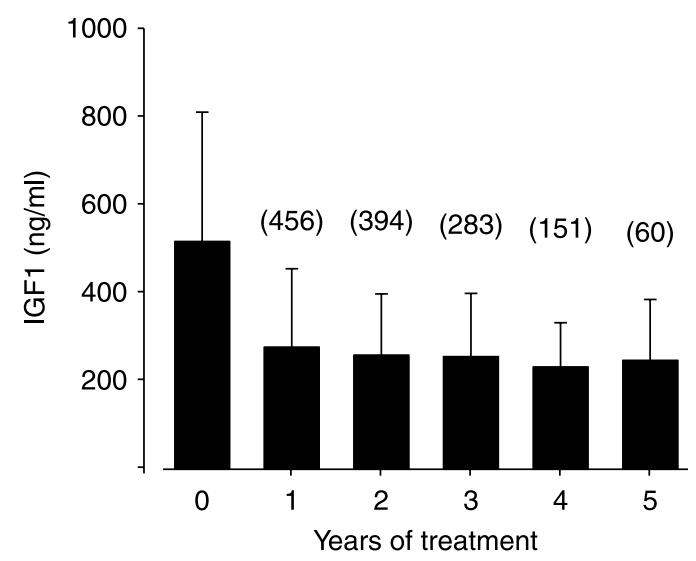

Figure 1 Serum IGF1 levels prior to and annually for the first 5 years after commencing pegvisomant therapy. Patient numbers are shown in brackets.

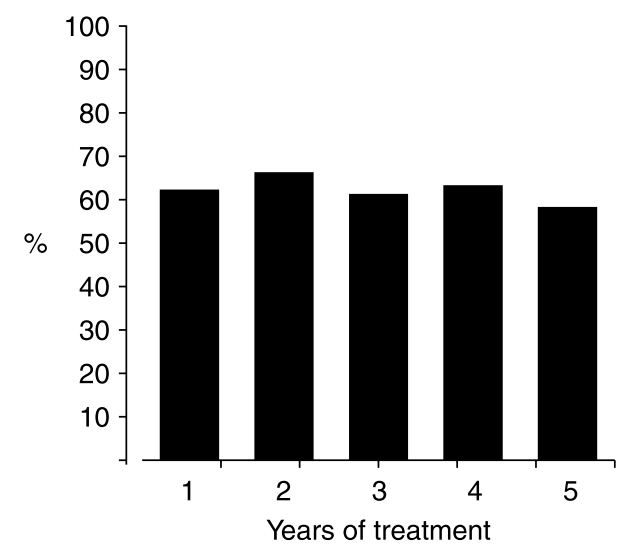

Figure 2 The percentage of patients with an IGF1 below the upper limit of the local age-related reference range.

The mean IGF1 prior to commencing pegvisomant was $518 \pm 296 \mathrm{ng} / \mathrm{ml}$ and after 1 year had fallen to $277 \pm 180 \mathrm{ng} / \mathrm{ml}$, remaining constant thereafter (Fig. 1). One year after the start of pegvisomant treatment, serum IGF1 was less than the upper limit of age-related reference range normalisation in $62 \%$ and remained constant for the 4 years thereafter (Figs 1 and 2).

As a proportion of patients were on weekly pegvisomant, dosing data are expressed as total dose per week regardless of dosing regime. One year after entry to ACROSTUDY, the mean weekly dose of pegvisomant in patients with a normal IGF1 was 106 and $113 \mathrm{mg}$ in those with an elevated IGF1, there being a trend for patients with an elevated IGF1 persisting on a slightly larger dose of pegvisomant (Fig. 3). However, many patients, despite elevated IGF1 levels, remained on relatively modest doses of pegvisomant (Fig. 4).

About $67 \%$ of patients entering ACROSTUDY were on pegvisomant as monotherapy, $6 \%$ were receiving pegvisomant along with a dopamine agonist and $23 \%$ a somatostatin analogue. Pegvisomant was used in combination with a somatostatin analogue and

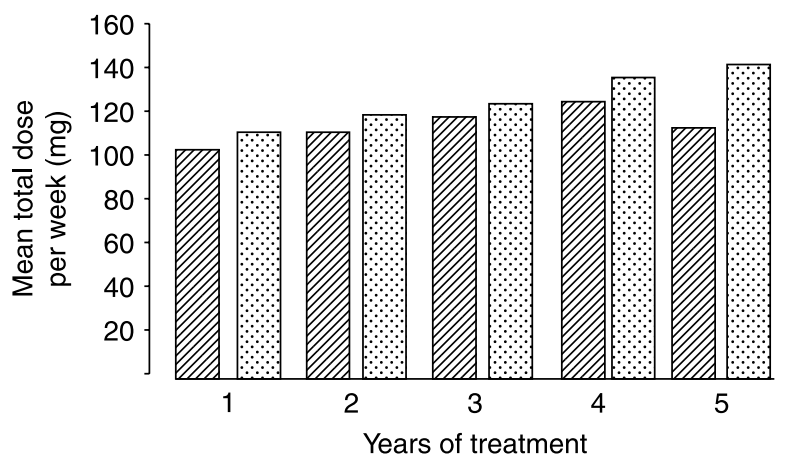

Figure 3 The mean total weekly dose of pegvisomant, expressed as a weekly dose as some patients were on less than daily dosing. Elevated IGF1, 巡 normal IGF1. 


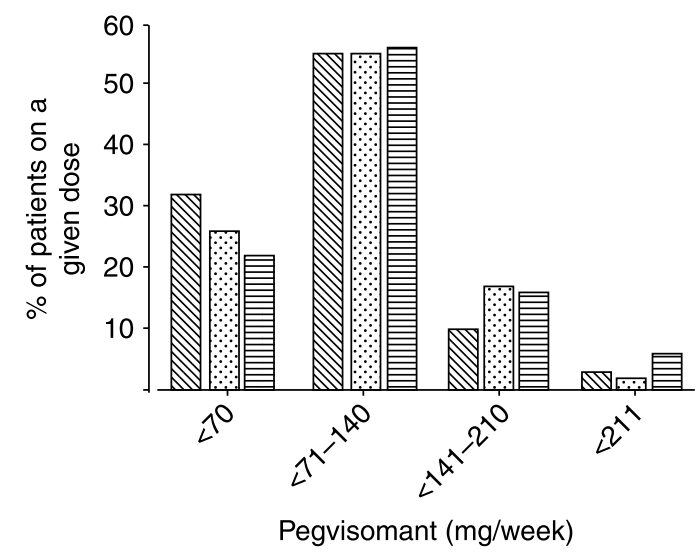

Figure 4 Distribution of total weekly dose in patients with persisting elevation of IGF1. Year 1 瓜, year 2 圈, year 3 目.

dopamine agonist in $4 \%$ of patients. The rate of IGF1 normalisation remained the same regardless of whether pegvisomant was used alone or in combination with a somatostatin analogue or dopamine agonist (Fig. 5).

\section{Safety analysis}

A total of 56 serious AEs (SAEs) were reported in 46 patients, of which 13 were attributed to pegvisomant, all but one (subarachnoid haemorrhage) either related to elevated liver enzymes $(n=9)$ or pituitary tumour growth $(n=3)$.

A total of 276 AEs have been reported in 142 patients (18\%), of which 56 non-SAEs were considered related to pegvisomant. The AEs most frequently attributed to pegvisomant were disturbed liver function tests and injection site reactions (Table 1).

The definition of abnormal liver enzymes was a $\geq 3$-fold elevation of least one of the enzymes $(\gamma$-glutamyl

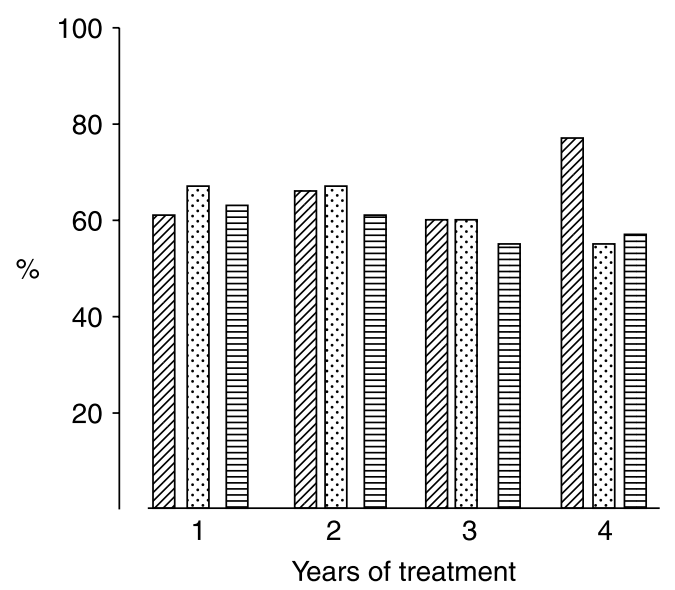

Figure 5 The percentage of patients with an IGF1 below the upper limit of the local age-related reference range subdivided by the mode of pegvisomant therapy. Pegvisomant $\mathbb{Z}$, pegvisomant + dopamine agonist $[$, pegvisomant and somatostatin analogue 目.
Table 1 A comprehensive list of serious adverse events reported in the first 5 years of ACROSTUDY, reported by the investigator as not being pegvisomant related. Number of patients affected is in brackets.

Deaths (2) - cause unknown, age 79 and 83, multiple co-morbidities Cholecystectomy (5)

Growth of tumour (7)

Knee problems

Epilepsy

Diabetes mellitus

Dizziness

Uncontrolled IGF1

Hip surgery

Pulmonary embolism

Pneumonia

Facial paralysis

Hypertension

Hypovolemic vertigo exsiccosis

Mitral valve insufficiency

Acute adrenal insufficiency crisis (2)

Arterial thrombosis at right leg

Cardiac dysrhythmia

Coprostasis

Coronary three vascular CHD with PTCA and stent

Due to infection, aggravation of COPD

Hospitalisation due to worsening of general condition

Laboratory abnormalities

Malignancy (5)

Total oophrectomy: haemorrhagic follicular cyst

Tentorial meningioma

transferase, AST and ALT). A total of 29 patients (3.7\%) had elevated liver enzymes during follow up, including cases in which it occurred before commencing pegvisomant. In 10 patients, the liver enzymes normalised; in five patients, this occurred despite continuing pegvisomant and in another five patients, pegvisomant was discontinued, although subsequently restarted in four. At data closure, liver function had not been documented as returning to normal in 16 patients; in three patients, liver enzymes were reported only once, and in two patients, liver enzymes had improved but not truly normalised by the last study visit. There is no evidence of sustained liver damage in any patient on pegvisomant.

Magnetic resonance imaging (MRI) was available in 684 patients. A total of 411 patients had at least one MRI on pegvisomant available for comparison with a pre-treatment scan with the longest duration of MRI follow-up being 8.9 years. Change in tumour size has been reported in 70 patients. In 31 patients, a decrease in tumour size has been documented, of whom 18 had previously received radiotherapy and eight were on pegvisomant plus a second agent (two somatostatin analogue, one somatostatin analogue plus a dopamine agonist and five a dopamine agonist alone). An increase in tumour size has been suspected and confirmed in 22 patients, of whom six had previously had radiotherapy and two had stopped a somatostatin analogue. The increase in tumour size occurred in two patients before commencing pegvisomant. In 11 patients, contradictory data exist on tumour size change, while, 
Table 2 A comprehensive list (in alphabetic order) of adverse events regarded by the reporting investigator as not being pegvisomant related. Number of patients affected is in brackets.

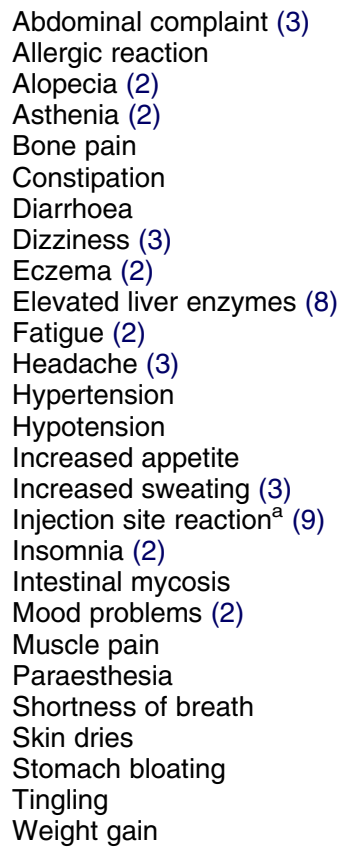

Abdominal complaint (3)

Allergic reaction

Alopecia (2)

Asthenia (2)

Bone pain

Constipation

Diarrhoea

Dizziness (3)

Eczema (2)

Elevated liver enzymes (8)

Fatigue (2)

Headache (3)

Hypertension

Hypotension

Increased appetite

Increased sweating (3)

Injection site reaction ${ }^{\mathrm{a}}(9)$

Insomnia (2)

Intestinal mycosis

Mood problems (2)

Muscle pain

Paraesthesia

Shortness of breath

Skin dries

Stomach bloating

Tingling

Weight gain

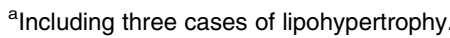

in patients, central review of the films failed to confirm the increase in tumour size suspected by the investigator.

Local injection site reactions have been reported in nine cases, of which three are known to be lipohypertrophy (Table 2).

There have been two deaths, but there is nothing in the information available to suggest a relationship to pegvisomant and, otherwise, there have been no obvious unforeseen pegvisomant-related AEs.

\section{Discussion}

The initial placebo-controlled, randomised trial of pegvisomant demonstrated normalisation of IGF1 in $90 \%$ of patients treated with $20 \mathrm{mg} /$ day, while the openlabel extension study reported normalisation in $97 \%$ of patients treated with doses up to $40 \mathrm{mg} /$ day $(1,2)$. It is therefore a disappointment that in ACROSTUDY, IGF1 normalised in $<70 \%$ of patients (Figs 1 and 2 ). Several factors probably contributed to the apparently disappointing efficacy in observed practice. In the clinical trials, IGF1 normalisation was defined as an IGF1 within the reference range at any stage during the trial, which contrasts to the cross-sectional presentation of data from ACROSTUDY. Particularly with the challenges of IGF1 assays, it is likely that some patients had, at previous visits, an IGF1 within the upper limit of the reference range, but because of inter-assay variation the data point included may have been above the upper limit of the reference range (11).

However, the principal reason for the disappointingly low rate of IGF1 normalisation appears to reflect a failure to adequately increase the dose of pegvisomant. Three years into therapy, $\sim 80 \%$ of patients with an elevated IGF1 are still on an average daily dose of $20 \mathrm{mg}$ or less (Figs 3 and 4). It takes 4-6 weeks for serum IGF1 to reach a steady state after a dose change and, with dose increments of $5 \mathrm{mg}$, it should be possible to reach a dose that controls the great majority of patients within a year. The nature of ACROSTUDY does not allow identification of the reasons for the failure of dose titration, but several factors may influence the decision not to increase the dose of pegvisomant until the desired biochemical goal is achieved. There may be a loss of motivation on the patient's and/or physician's part to further dose titrate, once a patient's symptoms have resolved. This may be compounded by a reluctance to accept the evidence that it is necessary to lower IGF1 to within the reference range to restore life expectancy to normal $(12,13)$. A further factor may be the linear pricing of pegvisomant, which means that the cost associated with dose escalation may be perceived as inhibitory. As with any medication, poor compliance may be a contributor, which could be studied by monitoring plasma pegvisomant concentration, although to date no data are available. An additional factor discouraging dose titration may be that a dose of $>20 \mathrm{mg}$ requires more than one vial and an injection volume of $>1 \mathrm{ml}$. Further efforts are required to understand and address the disappointing rate of IGF1 normalisation with pegvisomant therapy administered outside of clinical studies, and, in particular, the reasons for the failure of dose escalation need to be explored. Poor compliance could be addressed by less frequent dosing of pegvisomant, such as weekly doses (14).

In Europe, pegvisomant is indicated for use in patients unresponsive or intolerant to somatostatin analogues. It is a major point of debate whether in such circumstances pegvisomant should be added to ongoing somatostatin analogue treatment or alternatively substituted for the somatostatin analogue. A series of reports from van der Lely's group have demonstrated that the addition of once weekly pegvisomant to somatostatin analogue treatment normalises IGF1 in virtually every patient, and have furthermore suggested that the combination of weekly pegvisomant plus a somatostatin analogue could be up to $€ 58000$ less expensive than pegvisomant monotherapy $(15,16)$. In the only controlled study of patients with persistent elevation of IGF1 on long-acting octreotide, patients were randomised either to pegvisomant in combination with ongoing octreotide therapy or alternatively to stop octreotide and commence daily pegvisomant. There was no difference in the IGF1 normalisation rate, with patients on monotherapy requiring on average $5 \mathrm{mg}$ more pegvisomant per day than those on combination 
treatment, which indicates that the decision to use pegvisomant as monotherapy or in combination is cost neutral (17). The data from ACROSTUDY reinforce the notion that the biochemical control achieved is not different if pegvisomant is used as monotherapy or in combination with a somatostatin analogue (Fig. 5), suggesting that the decision whether to use pegvisomant as monotherapy or in combination should be based on an individual patient's characteristics. Significant tumour shrinkage with a somatostatin analogue argues for the addition of pegvisomant, while in a patient in whom glucose tolerance deteriorated with octreotide, pegvisomant monotherapy may be appropriate (18-20).

The mechanism of the disturbed liver function tests reported with pegvisomant remains poorly understood. In ACROSTUDY as with any large cohort of patients, the disturbance in liver function tests is likely to be of mixed aetiology. In some patients, liver function was already disturbed on entry to ACROSTUDY even before receiving pegvisomant, while, in other patients, it was documented on only one occasion and resolved despite continuation of pegvisomant, in one case even despite an increase in dose. There is compelling evidence that patients are at risk of gallstone-related problems in the months following cessation of somatostatin analogues, which may coincide with initiation of pegvisomant and account for some case of disturbed liver function tests (21). However, it must be acknowledged that in some patients, a very dramatic elevation in liver enzymes is seen in the early months after commencing pegvisomant. Detailed studies, including a liver biopsy of one patient, showed a chronic mild hepatitis with a mixed portal inflammation including eosinophilic granulocytes. The gold standard for confirmation of pegvisomant as the aetiology is resolution on discontinuation and recurrence on rechallenge (7). The incidence of pegvisomant-related disturbance of liver function tests is probably $<3 \%$, and reassuringly there is no evidence of persisting hepatic damage in any patients. In the coming years, ACROSTUDY should provide assistance in clarifying the incidence and natural history. Pegvisomantrelated liver function disturbance appears to occur within months of initiation, and it is important to regularly monitor liver function during the first 6 months and when subsequently monitoring IGF1 (18).

Injection site reactions fall into two categories, either atopic reactions or lipohypertrophy, the consistency of the latter with previous reports may be explained by profound $\mathrm{GH}$ resistance in proximity to the site of pegvisomant injection with the resultant unopposed action of insulin leading to lipohypertrophy (22-24). Rotation of the injection site may assist in reducing the likelihood of developing injection site reactions.

The data from ACROSTUDY on changes in tumour size are generally reassuring and consistent with the experience from other sources (4). The reduction in tumour size reported in some patients is presumably a reflection of prior radiotherapy. In three patients, investigators reported tumour growth as a pegvisomant-related SAE, but in the majority of patients with tumour expansion, the investigators did not judge it to be treatment related. In six patients, central re-evaluation of sequential MR scans was able to offer reassurance that there had not been an expansion in tumour size. This facility within ACROSTUDY is open to all investigators and will hopefully be called upon with increasing frequency. The limited number of patients and duration of therapy mean that definitive conclusions cannot be drawn, but the hope is that accumulation of additional experience will allow determination of whether or not there is any risk of tumour growth attributable to pegvisomant.

While the experience captured by ACROSTUDY remains modest compared with other similar registries such as KIMS for patients with GH deficiency, with time, it will answer not only questions about the long-term safety and tolerability of pegvisomant but also about the impact of vigorous control of IGF1 on the natural history of acromegaly.

\section{Declaration of interest}

I have served on the supervisor board of ACROSTUDY, which is sponsored by Pfizer. I have received lecture and consultancy fees plus research grants from Pfizer. I have received research grants, lecture fees, honoraria, and advisory council status (ACROSTUDY) with Pfizer with regards to Pegvisomant. ACROSTUDY ${ }^{\mathrm{TM}}$ is sponsored by Pfizer Inc. This paper forms part of a European Journal of Endocrinology supplement, supported by Pfizer Inc.

\section{Acknowledgements}

The assistance of Frida Lundgren with data analysis is gratefully acknowledged.

\section{References}

1 Trainer PJ, Drake WM, Katznelson L, Freda PU, Herman-Bonert V, van der Lely AJ, Dimaraki EV, Stewart PM, Friend KE, Vance ML, Besser GM, Scarlett JA, Thorner MO, Parkinson C, Klibanski A, Powell JS, Barkan AL, Sheppard MC, Malsonado M, Rose DR, Clemmons DR, Johannsson G, Bengtsson BA, Stavrou S, Kleinberg DL, Cook DM, Phillips LS, Bidlingmaier M, Strasburger CJ, Hackett S, Zib K, Bennett WF \& Davis RJ. Treatment of acromegaly with the growth hormone-receptor antagonist pegvisomant. New England Journal of Medicine 200020 1171-1177.

2 van der Lely AJ, Hutson RK, Trainer PJ, Besser GM, Barkan AL, Katznelson L, Klibanski A, Herman-Bonert V, Melmed S, Vance ML, Freda PU, Stewart PM, Friend KE, Clemmons DR, Johannsson G, Stavrou S, Cook DM, Phillips LS, Strasburger CJ, Hackett S, Zib KA, Davis RJ, Scarlett JA \& Thorner MO. Long-term treatment of acromegaly with pegvisomant, a growth hormone receptor antagonist. Lancet $20013 \mathbf{3 5} 1754-1759$.

3 Veldhuis JD, Bidlingmaier M, Anderson SM, Wu Z \& Strasburger CJ. Lowering total plasma insulin-like growth factor I concentrations by way of a novel, potent, and selective growth hormone (GH) receptor antagonist, pegvisomant (B2036-peg), augments the 
amplitude of GH secretory bursts and elevates basal/nonpulsatile GH release in healthy women and men. Journal of Clinical Endocrinology and Metabolism 200186 3304-3310.

4 Jimenez C, Burman P, Abs R, Clemmons DR, Drake WM, Hutson KR, Messig M, Thorner MO, Trainer PJ \& Gagel RF. Follow-up of pituitary tumor volume in patients with acromegaly treated with pegvisomant in clinical trials. European Journal of Endocrinology 2008159 517-523.

5 Feenstra J, van Aken MO, de Herder WW, Feelders RA \& van der Lely AJ. Drug-induced hepatitis in an acromegalic patient during combined treatment with pegvisomant and octreotide long-acting repeatable attributed to the use of pegvisomant. European Journal of Endocrinology 2006154 805-806.

6 Bernabeu I, Cameselle-Teijeiro J, Casanueva F \& Marazuela M. Pegvisomant-induced cholestatic hepatitis with jaundice in a patient with Gilbert's syndrome. European Journal of Endocrinology $2009160869-872$.

7 Biering H, Saller B, Bauditz J, Pirlich M, Rudolph B, Johne A, Buchfelder M, Mann K, Droste M, Schreiber I, Lochs H, Strasburger CJ \& German Pegvisomant Investigators. Elevated transaminases during medical treatment of acromegaly: a review of the German pegvisomant surveillance experience and a report of a patient with histologically proven chronic mild active hepatitis. European Journal of Endocrinology 2006154 213-220.

8 Schreiber I, Buchfelder M, Droste M, Forssmann K, Mann K, Saller B, Strasburger CJ \& the German Pegvisomant Investigators. Treatment of acromegaly with the GH receptor antagonist pegvisomant in clinical practice: safety and efficacy evaluation from the German Pegvisomant Observational Study. European Journal of Endocrinology 2007156 75-82.

9 Strasburger CJ, Buchfelder M, Droste M, Mann K, Stalla GK, Saller B \& German Pegvisomant Investigators. Experience from the German pegvisomant observational study. Hormone Research 2007 68 70-73.

10 Brue T. ACROSTUDY: status update on 469 patients. Hormone Research 200971 34-38.

11 Bidlingmaier M. Pitfalls of insulin-like growth factor I assays. Hormone Research 200971 30-33.

12 Swearingen B, Barker FG II, Katznelson L, Biller BM, Grinspoon S, Klibanski A, Moayeri N, Black PM \& Zervas NT. Long-term mortality after transsphenoidal surgery and adjunctive therapy for acromegaly. Journal of Clinical Endocrinology and Metabolism 199883 3419-3426.

13 Holdaway IM, Rajasoorya RC \& Gamble GD. Factors influencing mortality in acromegaly. Journal of Clinical Endocrinology and Metabolism 200489 667-674.

14 Higham CE, Thomas J, Bidlingmaier M, Drake WM \& Trainer PJ. Successful use of weekly pegvisomant administration in patients with acromegaly. European Journal of Endocrinology 2009161 21-25.
15 Feenstra J, de Herder WW, ten Have SM, van den Beld AW, Feelders RA, Janssen JA \& van der Lely AJ. Combined therapy with somatostatin analogues and weekly pegvisomant in active acromegaly. Lancet $20053651644-1646$.

16 Neggers SJ, van Aken MO, Janssen JA, Feelders RA, de Herder WW \& van der Lely AJ. Long-term efficacy and safety of combined treatment of somatostatin analogues and pegvisomant in acromegaly. Journal of Clinical Endocrinology and Metabolism $2007924598-4601$.

17 Trainer PJ, Ezzat S, D'Souza GA, Layton G \& Strasburger CJ. A randomized, controlled, multicentre trial comparing pegvisomant alone with combination therapy of pegvisomant and long-acting octreotide in patients with acromegaly. Clinical Endocrinology, 2009. In Press DOI: 10.1111/j.1365-2265. 2009.03620.x.

18 Barkan AL, Burman P, Clemmons DR, Drake WM, Gagel RF, Harris PE, Trainer PJ, van der Lely AJ \& Vance ML. Glucose homeostasis and safety in patients with acromegaly converted from long-acting octreotide to pegvisomant. Journal of Clinical Endocrinology and Metabolism 200590 5684-5691.

19 Rose DR \& Clemmons DR. Growth hormone receptor antagonist improves insulin resistance in acromegaly. Growth hormone and IGF Research 200212 418-424.

20 Drake WM, Rowles SV, Roberts ME, Fode FK, Besser GM, Monson JP \& Trainer PJ. Insulin sensitivity and glucose tolerance improve in patients with acromegaly converted from depot octreotide to pegvisomant. European Journal of Endocrinology 2003149 521-527.

21 Paisley AN, Roberts ME \& Trainer PJ. Withdrawal of somatostatin analogue therapy in patients with acromegaly is associated with an increased risk of acute biliary problems. Clinical Endocrinology $200766723-726$.

22 Maffei P, Martini C, Pagano C, Sicolo N \& Corbetti F. Lipohypertrophy in acromegaly induced by the new growth hormone receptor antagonist pegvisomant. Annals of Internal Medicine 2006 $145310-312$

23 Marazuela M, Daudén E, Ocón E, Moure D \& Nattero L. Pegvisomant-induced lipohypertrophy: report of a case with histopathology. Annals of Internal Medicine 2007147 741-743.

24 Bonert VS, Kennedy L, Petersenn S, Barkan A, Carmichael J \& Melmed S. Lipodystrophy in patients with acromegaly receiving pegvisomant. Journal of Clinical Endocrinology and Metabolism 2008 $933515-3518$

Received 21 July 2009

Accepted 30 July 2009 